\title{
AN EXPLORATION OF GENDER BIAS IN INFORMATION TECHNOLOGY JOB ADVERTISEMENTS
}

\author{
Jennifer L. Breese, Penn State University, jzb545@psu.edu \\ Mauri Conforti, Geisinger Health Systems, maconforti1@geisinger.edu \\ Alan Peslak, Penn State University, arp14@psu.edu
}

\begin{abstract}
Research indicates the existence of gender bias in job advertisements (Bem \& Bem, 2006; Pedriana, 2004). Furthermore, Gaucher et al. (2011) found that job advertisements typically include more masculine-biased words that may discourage female applicants. A more current, technology-specific research effort may suggest otherwise. A data analysis of 1500 job descriptions obtained from a February 2020 dataset spanning three specific information technology job titles found no gender bias overall or in the selected job titles: Cybersecurity Analyst, Programmer Analyst or Systems Analyst. Additionally, 22,000 technology jobs were analyzed based on a dataset obtained from Dice.com. The analysis found that, overall, job descriptions in information technology contained more feminine than masculine words; thus, indicating no male gender bias. While there is significant data to support the assertion that the information technology field is vastly underrepresented by females, there is no evidence to support the affirmation that masculine-coded job advertisements contribute to the underrepresentation of women in information technology.
\end{abstract}

Keywords: Gender Bias, Women in Information Technology, Job Advertisement Wording

\section{INTRODUCTION}

This paper explores information technology job advertisements to determine whether gender bias exists, either implicitly or explicitly, in the advertisements' wording. Bias results in unfair or harmful impact on people, such as relying on stereotypes based on another person's race, gender, ethnicity or religion to judge them, and a large body of research affirms that when differential rates and levels of bias exist, diversity of knowledge, experience, and background are irrevocably lost. Seemingly, a strong factor or group of factors negatively influence and discourage women from pursuing computing degrees and computing professional jobs. Though there are many reasons for the gender disparity, one leading issue may be biased language that favors males in job advertisements through the use of “masculine” words and phrases (Gaucher et at., 2011). Job parity between men and women has been a long- standing issue in myriad career fields. The typical perception in the technology sector suggests a tremendous lag in equal opportunities for men and women. In particular, information technology jobs remain heavily skewed toward males; however, other STEM careers, such as science, engineering, and mathematics have seen a significant balancing of women and men in the professions (Cruickshank, 2018; Gaucher et al., 2011). In this study, a semiotic framework was applied to the work of Gaucher et al. (2011) that explored gender bias in online job postings in addition to the analysis of two datasets to examine the wording of current information technology job advertisements. The current study was conducted to further discern whether biased language is still as common in IT job postings as it was in 2011. The goals of the current study were to learn whether language in job postings may account for the low representation of women in computer and other IT occupations and to determine if the language may perpetuate the ongoing disparity.

Notably, the balance occurring in other STEM fields does not extend to women in computer science whose numbers have steadily declined since 1991 (Williams, 2015). According to the National Science Foundation (2017), 47\% of all science jobs are currently held by women. However, the National Center for Women in Information Technology (NCWIT) found a widening gender disparity in information technology jobs. Additionally, the percentage of women earning computing degrees has steadily declined since its peak in 1985 when $37 \%$ of computer science bachelor's degrees were awarded to women. By 2017, the number had dropped dramatically to just $19 \%$ (Women in data science, 2020). In 2018, women held 57\% of all professional occupations; however, women held only $26 \%$ of professional computing occupations and only $20 \%$ of CIO positions (NCWIT, 2020). The STEM fields require a high education 


\section{Issues in Information Systems}

Volume 21, Issue 3, pp. 189-199, 2020

level for job entry (Graf et al., 2018), which has propelled others to explore the existence of bias embedded within the educational process (Herbert, 2013) and the workplace (Beasley, 2017).

\section{Bias and diversity}

Unconscious bias, sometimes called implicit bias, indicates a lack of conscious awareness. Unconscious bias leads to changes in thoughts, feelings and beliefs about the world we live in and also leads to cognitive bias or mistakes in a cognitive process, such as remembering, reasoning, evaluating or decision making. These unconscious biases occur because people tend to hold their own preferences and beliefs as true, despite contradictory evidence (Greenwald \& Krieger, 2006). Patterns for bias mainly function as implicit bias that reflect stereotypes people do not realize they possess; however, recent evidence suggests explicit bias based on race is alive and well (Williams 2015). Diversity programming created to confront bias in industry has seemingly failed to promote equality (Dobbin \& Kalev, 2016). Forced rather than voluntary diversity training might actually be to blame for promotion and hiring decisions. Forcing training rather than forcing simple social accountability by asking for explanations on employment hiring decisions might create an environment for better diversity and lesson the likelihood of actions rooted in bias (Dobbin \& Kalev, 2016).

Businesses started to care more about diversity after a number of high-profile lawsuits shook the financial industry; however, using the same programs from the 1960's could make things worse instead of better (Dobbin \& Kalev, 2016). In the last few decades, Americans in 22 states have filed lawsuits for employment protections on the basis of sexual orientation-based gender identity discrimination; protections have only been enacted in 19 states. Apple CEO Tim Cook advocated for the adoption of the Federal Employment Discrimination Act (ENDA) in 2013, but Congress has failed to act. Meanwhile, research has proven that corporate innovation is positively impacted through increased diversity (Gao \& Zang, 2016). Similarly, Apfelbaum (2016) found verbiage in company's diversity statements to be a factor in successful diversity within the company. The ways companies talk about diversity in their statements was a proxy for how they approach diversity. Generally, wording with a focus on 'value differences' equated to less attrition than 'equality,' especially among women. The difference in wording prompts a question about whether women are reading the diversity statements prior to application submission, thus delving deeper into potential company culture, in addition to (or separate from) job advertisements. Technology companies too have consistently allocated money and supportive rhetoric into annual diversity initiatives, but fail to both see and celebrate the benefits diversity can yield (Newkirk, 2019).

The steady decrease of women in computer science has been attributed to a need for greater work-life balance rather than gender bias (Williams, 2015). Yet, anecdotal insights from women leaving the field cannot be ignored. Patterns suggest that women felt a need to behave in ways that were more masculine to be seen as competent, and women seemingly walked a tightrope between being 'likeable' (feminine) or 'capable' (masculine) (Williams, 2015). Only one in six firms hold managers accountable to promote women or other minorities in the workplace. Despite the lack of regulatory requirements from the government, or a push from social movements, firms need to find accountability among their own ranks (Stainback \& Tomaskovic-Devy, 2013). Further data from Stainback and Tomaskovic-Devy (2013) indicates that equal opportunity has stalled since the Civil Rights Act of 1964, and many firms now demonstrate increased racial and gender segregation.

\section{Gender literature}

Cejka and Eagly (1999) performed an oft-cited study of gender stereotypes in over 80 occupations and found that the beliefs of the individuals surveyed mapped gender stereotyped attributes to actual gender placements in specific occupations. Therefore, if study participants saw feminine physical, personality, or cognitive traits as essential to success, then the traits mapped the incidence of primarily females in those occupations. Cejka and Eagly (1999) also confirmed a male gender bias in occupations with higher prestige and those seemed to require masculine personality and cognitive attributes. Higher earnings were also associated with male personality attributes. Male personality attributes included traits described as competitive, dominant, and courageous. Masculine physical attributes included items described as vigorous and strong. Male cognitive attributes included analytical, exact, and good at abstractions. 


\section{Issues in Information Systems}

Volume 21, Issue 3, pp. 189-199, 2020

The stereotypes vary with exposure to advertising and media, culture and society, an also exist in classrooms, which affects gender equity in education. The stereotyping is evident in advertisements about the technology industry. While it may appear that gender-specific job tasks have little or no bias, subtle biases are still prevalent. The proliferation and use of personal computers have given females more access to technology. Yet, advertisers still portray females in lesser roles or as being sexually attractive, while males have more dominant roles. Stereotypical males tend to use powerful statements, such as "work smarter not harder," "gain the recognition you deserve," and "take charge," while stereotypical females use phrases, such as "when you need to call home," and "I know how you feel." (Knupfer, 1998)

Females in grades kindergarten through high school showed less interest in advanced level computing classes; therefore, fewer women pursued computer-related degrees in college. The decline seemed to begin at the elementary education level. Moreover, at the high school level, only $12 \%$ of female students enrolled in computer electives, and only $17 \%$ of the female students participated in the advanced placement computer exam. In large part, girls who were computer savvy were perceived as nerds. This trajectory extends to the workforce. By 1994, women in the professional IT workforce accounted for $25 \%$ of the $46 \%$ of total working women. Several factors account for the low percentage, such as a lack of role models, sexism, and social conditioning that suggests computer-based technology careers are better intended for males. Similarly, males dominated the computer sales force, the cover of software packages, and advertising images (Verbick, 2002).

In 1996, toymaker Mattel released its Barbie Fashion Designer in an attempt to address gender disparity. Girls could use the Barbie Fashion Designer to design and print clothes for Barbie. Additional toys and software followed that was designed as either gender neutral or with girls as the primary users. This accessibility seemingly increased girls' interests in computer-based technology. In addition, websites emerged that promoted women in the computer science field and information about careers for women. (Verbick, 2002).

In 2010, the number of female and male students graduating from high school who were planning to pursue a career in science, technology and engineering was roughly equal. When students are told that both genders are equally capable, the difference in standardized test scores disappears. But stereotypes have proven detrimental to female students' desires to learn math and science skills. Fewer females than males pursue STEM careers, and women earn only $20 \%$ of the bachelor's degrees conferred in computer science, engineering and physics. (Hill et al., 2010).

Math and science job positions are more closely associated with males. Women in math and science job positions are more likely perceived negatively and as less competent. Competency perception only increases when the women are clearly successful in their work. Because likeability and competence are both seemingly necessary for career success, it can be difficult for females to promote to high-level job positions. In a 2009 poll, $74 \%$ of college-bound males and $32 \%$ of females were interested in computer science or computing as a major. Females believe they will have less success in these fields and, as a result, have less interest in pursuing those (Hill et al., 2010).

Interestingly, Tang et al. (2017) showed a decline in gender-biased job market postings over the last 10 years. Job listings often include subtle biases that discourage different candidates from applying for a position. Tang et al.'s (2017) study analyzed data obtained from 10 years of job postings on LinkedIn, user surveys from Amazon Turk workers, and undergraduate college students. The study indicated that gender-biased language in job postings has decreased in the last 10 years. Most masculine word frequency either remained consistent or decreased, while feminine words showed an increase in usage over time. Though gender neutral postings are more common, the perception in the user study showed they still feel the job market does not necessarily have gender neutrality. These gender biases deterred applicants from applying more frequently than the job posting wording. The gender perceptions, not the actual wording in the job positions, influences potential applicants and discourages them from applying. After removing the gendered language, the trend remained. The impact of gender-neutral job postings appears to have little effect in promoting a gender-neutral workforce. The applicants' inherent gender bias needs to be addressed to progress gender neutrality (Tang et. al., 2017).

\section{Semiotics}

A semiotic framework is used to assist in understanding why words, like actions, are contextually situated. Lacan (as cited in Buren, 1992) presupposed that individual women spoke and acted in the social sphere, but that in doing so, 


\section{Issues in Information Systems \\ Volume 21, Issue 3, pp. 189-199, 2020}

women submitted themselves to unconscious male structures. Further research suggested that women make a choice when entering male social structures, that women must reframe themselves into men. Consequently, women active in the public sphere struggle within a dichotomous relationship with their "true" nature, as the mother/fusion identity dominates gender definitions. French feminist theorists, Julia Kristeva (1980,1982,1984), Helene Cixous (1986), and Luce Irigaray (1985), argue that preverbal, presymbolic mental life cannot be split and separated from conscious discourse and life within the public culture must follow the traditional male pathways (Buren 1992). Hence, gender norms encapsulate culture and subsequent interactions within that culture (Buren, 1992). As a result, conducting a semiotic analysis of culturally-embedded texts is imperative to identify words and/or texts that imply gender meaning in order to determine the existence of gender bias that influences interactions and behaviors within a culture. In this study, information technology employment will be the culture analyzed through the lens of semiotics.

The term "semiotics" originates from the Greek word Seme (semiotikos) and means the study of signs, symbols and signification; moreover, semiotics is how meaning is created contextually, regardless of fact or fiction (Chandler, 2007; Eco, 1976; Radford, 2003) and is mainly concerned with signs as social forces. In broad terms, semiotics is a study of signs and a further definition extends meaning to signs. The volume of interdisciplinary material about semiotics amasses nearly 17,000 results on general Google and 690,000 Google Scholar.

Signs are not solely based on sign construction. Signs are also the objects of the theory of codes. Codes define content through a lens or fact-assuming unit like culture; defining culture in the context or lens of semiotics creates group mentality. Therefore, codes, or in this case words, created in job postings are the interpretant, not the interpreter; thus, codes determine meaning in the absence interpretation (Eco, 1976). Codes are often found in the workforce as a form of high context communication or jargon. Additionally, job postings are not void of cues because they may be riddled with other semiotic referents that indicate voice.

A precursor to the study of Semiotics is the science of symbolism. Ogden and Richards (1923) explored the science of symbols and the construction of meaning. They explained logistics and semiotics without complex laws of grammar. For Ogden and Richards (1923), semiotics is the study of signs they term the "science of symbolism." While the spoken word is the most important instrument in civilization, symbols and the relationship between words is paramount in linguistic studies. Ogden's and Richards's 1923 work, while more psychological in nature, developed the beginnings of the semiotic triadic approach to communication that emphasized the importance of the referent, or what the sign stands for ,in the semiotic relationship. The scholarly assertion is that semiotics represents a challenge to the literal because semiotics rejects the notion that humans can neutrally represent the way things are without interpretation by the mind or language. Humans are continually searching to assign meaning through words or signs. Various models and tools have emerged to better understand how meaning emerges or is created. Chandler (2007) analyzed two models: the Peircean Model by philosopher Charles S. Peirce, and the Saussurean Model developed by linguist Ferdinand de Saussure. Chandler (2007) concluded that many individual signifiers make up one sign, all meaning is derived by individual things, and all of these factors contribute to the meaning of the sign. Moreover, the "signifier" is the form or practice a sign takes or the sign in action. The "signified" is the reference to the reality that is created. Consequently, this study accepts the meaning generated by the relationship between the signifier and the signified based on the social and cultural conventions learned (Chandler, 2007).

In summary, humans think in terms of signs and are constantly seeking a sense of meaning. Each person's own determination of meaning is dependent on previous texts or codes based on past experience(s). When an uncertainty 'text' arises, a guess will ensue and a 'proper' meaning will be inserted if and when additional information is gathered. As a result, new language symbols are formed and their individual meanings are ingrained. Finally, and most importantly, the language symbols now create and signify meaning among groups, which is critical to identify whether gender bias occurs in a job posting.

\section{METHODOLOGY}

Using the same dictionary of masculine and feminine words Gaucher et al. (2011) identified, the study examined the recent state of gender bias in information technology job advertisements. The postjobfree.com website served as the 


\section{Issues in Information Systems \\ Volume 21, Issue 3, pp. 189-199, 2020}

data source. The data source differs from more familiar job posting sites, such as Indeed.com, because its terms of service have no restrictions and postings are $100 \%$ public. Indeed.com and other job sites have specific restrictions that prevent data extraction, and Postjobfree.com has no such restrictions (Postjobfree, 2020). Postjobfree.com allows a job title search and a query returns open jobs and descriptions. Specific job titles were extracted and examined in early 2020. Additionally, a 2017 dataset from Dice.com was analyzed that exclusively featured technology job postings. Gaucher et al. (2011) identified a measure of gendered wording created from lists of masculine and feminine words published alongside lists of agentic and communal words. The lists were developed based on extensive prior research, including: Bartz and Lydon (2004); Rudman and Kilianski (2000); Bem (1974); and, Schullo and Alperson (1984).

Sample: Three current and specific job titles were chosen for the searches: Cybersecurity, Programmer Analyst, and Systems Analyst. Postjobfree.com provides worldwide job postings but has a primary concentration in the United States.

Instrument: The tool used to categorize language, defined as words and phrases, in the job advertisements was based partly on Gaucher et al.'s (2011) methods. Gaucher et al. (2011) obtained a list of occupations with the proportion of women and men in each from the U.S. Department of Labor (2007) and then selected 11 occupations that were highly male- or female-dominated with a corresponding category on both job websites. They then selected words and phrases based on prior research deemed masculine-coded words and/or feminine-coded words. The list of masculine- and feminine-coded words is detailed in Appendix 1. The only change to the Gaucher et al. (2011) list was the exclusion of the word "nag," which is listed as a feminine word. "Nag" was excluded because it was unlikely to appear in any job ad and also the nag fragment as "nag" was recognized within the word management and the data sorts, thus potentially skewing the data.

Data Collection Procedures: Two researchers independently entered Cybersecurity Analyst, Programmer Analyst, and Systems Analyst as the search terms into Postjobfree.com and obtained 497 job descriptions for each job title. The queries were entered on February 17, 2020 and returned the maximum number of results permitted by the website.

In order to verify the results over a larger dataset, the researchers analyzed a 2017 dataset of technology jobs from Dice.com accessible through Kaggle. The 2017 dataset was publicly available. Though the dataset did not include usable job search categories, Dice.com is almost exclusively a technology online job site, and the dataset included 22,000 usable job descriptions to test for gender bias.

Data Analysis: The researchers utilized Excel to count instances of both masculine- and feminine-coded words based on the Gaucher et al. (2001) dictionary shown in Appendix 1. A variety of SPSS 26 tools was also used to determine whether the results were statistically significant.

\section{RESULTS}

This study examined whether a male bias, indicated using gender-biased language, is still inherent in current technology job advertisements. On average there were 3.78 instances of the masculine words per job ad and 4.60 of the feminine words per job ad based on a count from the dictionary (Table 1). The complete dictionary of words used is shown in Appendix 1. The difference between masculine and feminine words is significant at $\mathrm{p}<.001$. The current 1481 information technology job advertisements actually show a feminine bias. This finding is directly opposite the findings of Gaucher et al. (2011) and supports Tang et al. (2017), which showed a trend to more feminine coding and less gender bias. Results preliminarily suggest that current information technology-specific job advertisements do not include gender bias that favors male applicants.

Table 1. PostJobFree.com All Job Categories Paired Samples Statistics

\begin{tabular}{lll|l|l|l} 
& & & & $\begin{array}{l}\text { Std. } \\
\text { Mean }\end{array}$ \\
\hline Pair 1 & Male & 3.78 & $\mathrm{~N}$ & Std. Deviation & \\
\cline { 2 - 7 } & Female & 4.60 & 1481 & 2.254 & .059 \\
\hline
\end{tabular}




\section{Issues in Information Systems}

Volume 21, Issue 3, pp. 189-199, 2020

The analysis suggests the inherent male gender bias found by many previous studies seems to have been ameliorated. In fact, feminine words now appear far more frequently than masculine words, according to the more comprehensive measure. Also, the ads contained a total of 5591 masculine words compared to 6808 feminine words. This study analyzed the 500 job descriptions in each of the job categories to discern if there were significant gender bias differences based on the specific job title.

\section{Cybersecurity}

For cybersecurity analyst positions (Table 2), a significant number of feminine words existed compared to the masculine words. The difference was statistically significant at $\mathrm{p}<.001$. On average, the job postings contained 4.56 masculine words and 5.32 female words. Of all the job categories, cybersecurity analyst, surprisingly, had the greatest number of feminine words per the Gaucher dictionary.

Table 2. PostJobFree.com Cybersecurity Analyst

\begin{tabular}{lll|l|l|l} 
& & & & Std. \\
& & Mean & $\mathrm{N}$ & Std. Deviation & Mean \\
\hline \multirow{2}{*}{ Pair 1 } & Male & 4.56 & 497 & 2.496 & .112 \\
\cline { 2 - 6 } & Female & 5.32 & 497 & 2.575 & .115 \\
\hline
\end{tabular}

\section{Programmer Analyst}

Table 3 shows the results for programmer analyst. Again, more feminine words (4.18) appear versus masculine words (3.29). This is the highest gap between male and female words of the three jobs analyzed: cybersecurity analyst, programmer analyst, and systems analyst. Again, the difference is statistically significant at $\mathrm{p}<.001$.

Table 3. PostJobFree.com Programmer Analyst Paired Sample Test

\begin{tabular}{lll|l|l|l} 
& & & & $\begin{array}{l}\text { Std. } \\
\text { Mean }\end{array}$ \\
\hline \multirow{2}{*}{ Pair 1 } & Mean & $\mathrm{N}$ & Std. Deviation & \\
\cline { 2 - 7 } & Female & 3.29 & 495 & 1.934 & .087 \\
\hline
\end{tabular}

Finally, for systems analyst (Table 4) there were more feminine than masculine words and the results were significant at $\mathrm{p}<.0001$. There were 3.47 average masculine words and 4.28 feminine words.

Table 4. PostJobFree.com Systems Analyst

\begin{tabular}{lll|l|l|l} 
& & & & Std. & Error \\
& & Mean & $\mathrm{N}$ & Std. Deviation & Mean \\
\hline \multirow{2}{*}{ Pair 1 } & Male & 3.47 & 489 & 2.080 & .094 \\
\cline { 2 - 7 } & Female & 4.28 & 489 & 2.406 & .109 \\
\hline
\end{tabular}

As a last test of the Postjobfree.com dataset, the researchers analyzed whether there was a significant difference in the degree of feminine tone for each of the jobs in our dataset. An ANOVA test was performed and findings indicated no significant difference in the mean difference between male and female words in any of the jobs studied. The p value was .826.

Table 5. Postjobfree.com ANOVA test for differences between job titles

\begin{tabular}{ll|l|l|l|l} 
& $\begin{array}{l}\text { Sum } \\
\text { Squares }\end{array}$ & df & Mean Square & F & Sig. \\
\hline Between Groups & 4.002 & 2 & 2.001 & .191 & .826 \\
\hline Within Groups & 15492.938 & 1478 & 10.482 & & \\
\hline Total & 15496.940 & 1480 & & & \\
\hline
\end{tabular}




\section{Issues in Information Systems \\ Volume 21, Issue 3, pp. 189-199, 2020}

The results indicate several preliminary conclusions. First, male bias in job descriptions found by Gaucher et al. (2011) has been eradicated in information technology. Second, a small, but significant, female bias was identified in job advertisements seeking information technology workers. A limitation of the study was the small sample size. An attempt was made to remedy the deficiency by downloading and analyzing a large publicly available dataset $(22,000)$ of technology jobs from Dice.com to further identify the existence of a male gender bias in the ads. A limitation here was the 2017 date. As a result, the 2020 preliminary conclusions could not be directly verified; however, male or female bias was confirmed in the 2017 dataset. The expectation was the evidence of a significant bias in feminine versus masculine words, but that the bias would be less than the 2020 results. The 2020 results show total masculine words of 2.58 per ad versus 2.76 feminine words. The feminine bias was smaller than 2020, but it was still statistically significant at $\mathrm{p}<.001$. The statistical significance of $\mathrm{p}<.001$ supports preliminary findings that the male gender bias found in 2011 has been eliminated or at least dissipated in 2020.

Table 6. Dice.com Dataset

\begin{tabular}{lll|l|l|l} 
& & & & Std. & Error \\
& & Mean & $\mathrm{N}$ & Std. Deviation & Mean \\
\hline Pair 1 & $\begin{array}{l}\text { Total } \\
\text { M }\end{array}$ & 2.58 & 22000 & 2.055 & .014 \\
\cline { 2 - 6 } & TotalF & 2.76 & 22000 & 2.042 & .014 \\
\hline
\end{tabular}

Lastly, the researchers tested whether there was a statistically significant difference between any of the Postjobfree job postings and the Dice dataset with regard to gender bias. A significant difference did exist, but as the Post hoc analysis shows, the only differences were between the Dice dataset and the Postjobfree job titles.

ANOVA

Table 7 Dice vs. Postjobfree ANOVA

\begin{tabular}{ll|l|l|l|l} 
& $\begin{array}{l}\text { Sum } \\
\text { Squares }\end{array}$ & df & Mean Square & F & Sig. \\
\hline Between Groups & 563.058 & 3 & 187.686 & 47.756 & .000 \\
\hline Within Groups & 92267.620 & 23477 & 3.930 & & \\
\hline Total & 92830.678 & 23480 & & & \\
\hline
\end{tabular}

Table 8 Dice vs. Postjobfree Post hoc Analysis

Dependent Variable: MminusF

\begin{tabular}{|c|c|c|c|c|c|c|}
\hline \multirow[b]{3}{*}{ (I) V2 } & \multirow[b]{3}{*}{ (J) V2 } & \multirow{2}{*}{\multicolumn{3}{|c|}{ LSD }} & \multirow{2}{*}{\multicolumn{2}{|c|}{ 95\% Confidence Interval }} \\
\hline & & & & & & \\
\hline & & Mean Difference (I-J) & Std. Error & Sig. & Lower Bound & Upper Bound \\
\hline \multirow[t]{3}{*}{ Cyber } & ProgAnal & .126 & .126 & .316 & -.12 & .37 \\
\hline & SysAnal & .051 & .126 & .684 & -.20 & .30 \\
\hline & Dice & $-.576^{*}$ & .090 & .000 & -.75 & -.40 \\
\hline \multirow[t]{3}{*}{ ProgAnal } & Cyber & -.126 & .126 & .316 & -.37 & .12 \\
\hline & SysAnal & -.075 & .126 & .553 & -.32 & .17 \\
\hline & Dice & $-.702^{*}$ & .090 & .000 & -.88 & -.53 \\
\hline \multirow[t]{3}{*}{ SysAnal } & Cyber & -.051 & .126 & .684 & -.30 & .20 \\
\hline & ProgAnal & .075 & .126 & .553 & -.17 & .32 \\
\hline & Dice & $-.627^{*}$ & .091 & .000 & -.80 & -.45 \\
\hline \multirow[t]{3}{*}{ Dice } & Cyber & $.576^{*}$ & .090 & .000 & .40 & .75 \\
\hline & ProgAnal & $.702^{*}$ & .090 & .000 & .53 & .88 \\
\hline & SysAnal & $.627^{*}$ & .091 & .000 & .45 & .80 \\
\hline
\end{tabular}

* The mean difference is significant at the 0.05 level. 


\section{Issues in Information Systems}

Volume 21, Issue 3, pp. 189-199, 2020

\section{Discussion/Implications}

This study was an attempt to update and explore whether there has been a significant difference in gendered wording of information technology job advertisements from 2011 to present. Surprisingly, the findings seem to indicate male bias found in 2011 appears to no longer exist in 2017 or 2020. In fact, information technology job advertisements have changed so much that they may contain a feminine bias. Of course, further study of more job advertisements would be needed to confirm the findings, but the preliminary results seem compelling. This change in job advertisements, however, has not corresponded to a proportional increase in women in the information technology workforce. One barrier may have been removed, but much work remains in other areas to address the continued shortfall of female information technology students and professionals in the workforce.

\section{CONCLUSION}

While the numbers and results of this study appear promising based on the gender wording bias tool and an apparent shift toward feminine wording, further issues exist that require attention and exploration. Chiefly, future research could identify and examine other pressing issues that might account for the underrepresentation of women in the information technology workforce. Again, while the results of this study point to positive strides to eliminate bias and semiotic verbiage in information technology, Horsley (2020) found that women have lost their jobs at a much higher rate than men during the COVID-19 pandemic. Furthermore, Kalev (2016) found that layoffs disproportionately affect women and minorities, thus wiping out most of the gains made by only focusing on tenure and titles. Whereas performance appraisals could provide another avenue toward equality, they, too, show disproportionately lower scores for women and minorities (Kalev, 2016). Meanwhile. Loten (2020) brought to light the 112,000 job losses suffered in the information technology sector during the COVID-19 epidemic; but, the specificity of the job losses and potential gender weight or bias has yet to be reviewed or segmented. Ongoing research will be necessary to review information technology job positions held by women and the sustainability of those jobs during the COVID-19 global pandemic.

\section{REFERENCES}

Apfelbaum, E. (2016). Why your diversity program may be helping women but not minorities (or vice versa). Harvard Business School Cases, 1.

Bartz, J. A., \& Lydon, J. E. (2004). Close relationships and the working self-concept: Implicit and explicit effects of priming attachment on agency and communion. Personality and Social Psychology Bulletin, 30(11), 13891401. doi:10.1177/0146167204264245.

Beasley, M. (2017, March 29). There is a supply of diverse workers in tech, so why is Silicon Valley so lacking in diversity? Retrieved June 14, 2018, from https://www.americanprogress.org/issues/race/reports/2017/03/29/429424/supply-diverse-workers-techsilicon-valley-lacking-diversity.

Bem, S. L. (1974). The measurement of psychological androgyny. Journal of Consulting and Clinical Psychology, 42(2), 155-162. https://doi.org/10.1037/h0036215.

Bem, S. L., \& Bem, D. J. (2006, July 31). Does sex-biased job advertising "aid and abet" sex discrimination? Retrieved May 19, 2020, from https://onlinelibrary.wiley.com/doi/abs/10.1111/j.1559-1816.1973.tb01290.x.

Bem, S. L., \& Bem, D. J. (1973, March). Does sex-biased job advertising "aid and abet" sex discrimination? Journal of Applied Psychology, 3(1), 6-18. https://doi.org/10.1111/j.1559-1816.1973.tb01290.x.

Buren, J. V. (1992). The semiotics of gender. Journal of the American Academy of Psychoanalysis, 20(2), 215-232. doi: 10.1521/jaap.1.1992.20.2.215. 
Cejka, M. A., \& Eagly, A. H. (1999). Gender-stereotypic images of occupations correspond to the sex segregation of employment. Personality and Social Psychology Bulletin, 25(4), 413-423.

Chandler, D. (2003). Semiotics: the basics. New York and London: Routledge. (SB)

Cruickshank, H. (2018, April 19). Women doctors vs. men doctors. Retrieved May 18, 2020, from https://www.healthline.com/health-news/women-doctors-better-patient-interactions.

Dobbin, F., \& Kalev, A. (2016). Why diversity programs fail. (cover story). Harvard Business Review, 94(7/8), 52-60.

Eco, U. (1976). A theory of semiotics. Bloomington: Indiana University Press.

Equality Challenge Unit (2013, September). Unconscious bias and higher education. Retrieved June 14, 2018, from https://www.ecu.ac.uk/wp-content/uploads/2014/07/unconscious-bias-and-higher-education.pdf.

Gao, H., \& Zhang, W. (2016, August 17). Non-discrimination laws make U.S. states more innovative. Retrieved May 8, 2020, from https://hbr.org/2016/08/non-discrimination-laws-make-us-states-more-innovative.

Graf, N., Fry, R., \& Funk, C. (2018, January 09). 7 facts about the STEM workforce. Retrieved June 14, 2018, from http://www.pewresearch.org/fact-tank/2018/01/09/7-facts-about-the-stem-workforce/.

Gaucher, D., Friesen, J., \& Kay, A. C. (2011). Evidence that gendered wording in job advertisements exists and sustains gender inequality. Journal of Personality and Social Psychology, 101(1), 109.

Greenwald, A., \& Krieger, L. (2006). Implicit bias: scientific foundations. California Law Review, 94(4), 945-967. doi:10.2307/20439056

Hill, C., St. Rose, A., \& Corbett, C. (2010). Why so few? Women in science, technology, engineering, and mathematics. Washington, DC: AAUW.

Horsley, S. (2020, April 8). Women are losing more jobs in coronavirus shutdowns. Retrieved May 10, 2020, from https://www.npr.org/2020/04/08/829141182/women-are-losing-more-jobs-in-coronavirus-shutdowns.

Kaggle (2017). Dice Dataset 2017. https://www.kaggle.com/salsitail55/dice-dataset?rvi=1.

Kalev, Alexandra. 2014. "How you downsize is who you downsize: Biased formalization, accountability, and managerial diversity.” American Sociological Review 79(1):109-135.

Knupfer, N. N. (1998). Gender divisions across technology advertisements and the www: Implications for educational equity. Theory into Practice, 37(1), 54-63.

National Science Foundation (2017, January). Women, minorities and persons with disabilities in science and engineering. Retrieved DATE from https://www.nsf.gov/statistics/2017/nsf17310/digest/fod-women.

Newkirk, P. (2019, October 10). The diversity business is booming, but what are the results? Retrieved May 8, 2020, from https://time.com/5696943/diversity-business/.

Ogden, C. K. \& Richards, I. A. (1923). The meaning of meaning: A study of the influence of language upon thought and of the science of symbolism. New York and London: Harcourt / HBJ Book Harcourt Brace Jovanovich.

Pedriana, N. (2004). Help wanted NOW: Legal resources, the women's movement, and the battle over sexsegregated job advertisements. Social Problems, 51(2), 182-201. doi:10.1525/sp.2004.51.2.182. 
Postjobfree.com. (2020). www.PostJobfree.com

Loten, A. (2020, May 8). Technology sector shed record number of jobs in April. Retrieved May 9, 2020, from https://www.wsj.com/articles/technology-sector-shed-record-number-of-jobs-in-april-11588974127.

Radford, G. (2003). On Eco. California: Wadsworth Publication Company.

Rudman, L. A., \& Kilianski, S. T. (2000). Implicit and explicit attitudes toward female authority. Personality and Social Psychology Bulletin, 26 (11),1315-1328.

Schullo, S. A., \& Alperson, B. L. (1984). Interpersonal phenomenology as a function of sexual orientation, sex, sentiment, and trait categories in long-term dyadic relationships. Journal of Personality and Social Psychology, 47(5), 983-1002. doi:10.1037/0022-3514.47.5.983

Stainback, K., \& Tomaskovic-Devey, D. (2013, June 19). Research: your firm probably isn’t an equal opportunity employer. Harvard Business Review, 122-127.

Tang, S., Zhang, X., Cryan, J., Metzger, M. J., Zheng, H., \& Zhao, B. Y. (2017). Gender bias in the job market: A longitudinal analysis. Proceedings of the ACM on Human-Computer Interaction, 1(CSCW), 1-19.

Terrell, J., Kofink, A., Middleton, J., Rainear, C., Murphy-Hill, E., Parnin, C., \& Stallings, J. (2017). Gender differences and bias in open source: Pull request acceptance of women versus men. PeerJ Computer Science, 3, e111.

Verbick, T. (2002). Women, technology, and gender bias. Journal of Computing Sciences in Colleges, 17(3), 240250.

Williams, J. C. (2017, December 6). The 5 biases pushing women out of STEM. Retrieved May 7, 2020, from https://hbr.org/2015/03/the-5-biases-pushing-women-out-of-stem.

Women in data science: The current gender diversity outlook. (2020, April 12). Retrieved May 19, 2020, from https://www.analyticsinsight.net/women-in-data-science-the-current-gender-diversity-outlook/. 
Issues in Information Systems

Volume 21, Issue 3, pp. 189-199, 2020

\section{Appendix A}

\begin{tabular}{|c|c|}
\hline Masculine words & Feminine words \\
\hline Active & Affectionate \\
\hline Adventurous & Child* \\
\hline Aggress* & Cheer* \\
\hline Ambitio* & Commit* \\
\hline Analy* & Communal \\
\hline Assert* & Compassion* \\
\hline Athlet* & Connect* \\
\hline Autonom* & Considerate \\
\hline Boast* & Cooperat* \\
\hline Challeng* & Depend* \\
\hline Compet* & Emotiona* \\
\hline Confident & Empath* \\
\hline Courag* & Feminine \\
\hline Decide & Flatterable \\
\hline Decisive & Gentle \\
\hline Decision* & Honest \\
\hline Determin* & Interpersonal \\
\hline Dominant & Interdependen* \\
\hline Domina* & Interpersona* \\
\hline Force* & Kind \\
\hline Greedy & Kinship \\
\hline Headstrong & Loyal* \\
\hline Hierarch* & Modesty \\
\hline Hostil* & Nag \\
\hline Implusive & Nurtur* \\
\hline Independen* & Pleasant* \\
\hline Individual* & Polite \\
\hline Intellect* & Quiet* \\
\hline Lead* & Respon* \\
\hline Logic & Sensitiv* \\
\hline Masculine & Submissive \\
\hline Objective & Support* \\
\hline Opinion & Sympath* \\
\hline Outspoken & Tender* \\
\hline Persist & Together* \\
\hline Principle* & Trust* \\
\hline Reckless & Understand* \\
\hline Stubborn & Warm* \\
\hline Superior & Whin* \\
\hline Self-confiden* & Yield* \\
\hline Self-sufficien* & \\
\hline Self-relian* & \\
\hline
\end{tabular}

Note. The asterisk denotes the acceptance of all letters, hyphens, or numbers following its appearance. 